\title{
Why do student attitudes toward science \& technology matter?
}

\section{Opinion}

Educational institutions, businesses, and political leaders are increasingly recognizing that scientific skills are necessary for economic opportunity and social mobility. Science continues to impact medicine, environment and numerous other sectors of society related to quality of life. Attitude toward a discipline is one of the major factors in students' choice of majors and careers. Pursuit of higher education in science and technology (S\&T) depends, to a great extent, on students' attitudes toward these disciplines. Consequently, the promotion of favorable attitudes toward science and technology, scientists and technologists, and learning about science and technology, is increasingly a matter of importance.

U.S. culture generally allows qualified students to more or less freely choose their fields of study, so rational major choice is largely determined by what those students value-that is, what they perceive to be worthwhile. Contemporary research in adolescent development has provided strong empirical support for the notion that personal assessments of value tend to guide choices. Plato (ca 380 BCE) pointed out about 2400years ago that ignorance can lead people to make mistakes in developing their values, so the obvious solution is education that eliminates the ignorance. Hence, one central task of legitimate S\&T recruitment is to educate a diverse set of academically talented students by providing them with sound reasons to value the study of technically-oriented fields enough to major in those fields, or to enroll in S\&T coursework.

A lack of diversity-including especially gender diversity-in the S\&T community has long been viewed as a critical problem, detracting from innovation and prosperity. For decades, researchers have attempted to explore in more detail the influence of gender stereotyping on college major choice; gender stereotyping is so deeply entrenched that it may not even be conscious. Among first-year college students, women are much less likely than men to say that they intend to major in S\&T. By graduation, men outnumber women in nearly every technical field. Women's representation in S\&T declines further at the graduate level, and yet again in the transition to the workplace. There is significant evidence that social and environmental factors contribute to the underrepresentation of women in S\&T. Structural obstacles like perception that there may not be equal opportunities for men and women to succeed in S\&T fields continue to dissuade women from pursuing a technically-oriented careers.

Our study designed and constructed a Scale to measure first-year college students' attitudes toward science and technology (S\&T), and specifically toward female participation in these disciplines. Results of data analyses showed that the Scale's overall reliability is high (0.85), and factor analyses revealed five orthogonal factors with high coefficient alphas-factors that represent relevant attitude constructs.

The five factors are:

a. Interest in gaining science and technology knowledge;
Volume I Issue I - 2017

\author{
Anu A Gokhale, Kenton F Machina \\ Department of Technology, Illinois State University, USA
}

Correspondence: Anu A Gokhale, Department of Technology, Illinois State University, USA, Tel 30943855 76,

Email aagokhale@ilstu.edu

Received: May 24, 2017 | Published: June 08, 2017

b. Science and technology are beneficial to humankind;

c. Appropriateness of science and technology for females;

d. Males and females have equal opportunity in science and technology;

e. Concern that science and technology are dangerous to humankind.

This Scale has been used by educators at the senior high school and college levels to evaluate the effectiveness of different teaching/ learning strategies in promoting positive student attitudes toward S\&T, and in improving learning among students. The Scale is unique in that it also includes attitudes toward gender equality of opportunity in S\&T. Our project has sought to influence and change students' attitudes by implementing "context-based" or Science/Technology/Society (STS) course content and visits with science and technology professionals in introductory general education coursework. We found these strategies to be successful in maintaining positive attitudes toward science and technology, especially among female college students, while in our control group female college student attitudes toward S\&T declined.

Countries that maintain a competitive edge and prosper will be countries that are most effective in developing their human capital and in nurturing individuals with the capabilities of developing new ideas and innovations, especially in the scientific and technological enterprise. The low participation of women in S\&T related careers is a matter of international concern. A lack of social and academic support for female students to pursue S\&T fields can impede their academic and career aspirations. The focus on reducing gender disparity across all fields of S\&T takes on greater urgency as we cannot afford to ignore the potential contributions of half of the world's population.

\section{Acknowledgements}

None.

\section{Conflict of interest}

Author declares that there is no conflict of interest. 